Chapman University

Chapman University Digital Commons

Sociology Faculty Articles and Research

8-20-2016

\title{
Decomposing Trends in Adult Body Mass Index, Obesity, and Morbid Obesity, 1971-2012
}

Ashley Wendell Kranjac

Robert L. Wagmiller

Follow this and additional works at: https://digitalcommons.chapman.edu/sociology_articles

Part of the Comparative Nutrition Commons, Human and Clinical Nutrition Commons, Medicine and Health Commons, Nutritional Epidemiology Commons, and the Other Nutrition Commons 


\section{Decomposing Trends in Adult Body Mass Index, Obesity, and Morbid Obesity, 1971-2012}

\section{Comments}

NOTICE: this is the author's version of a work that was accepted for publication in Social Science \& Medicine. Changes resulting from the publishing process, such as peer review, editing, corrections, structural formatting, and other quality control mechanisms may not be reflected in this document. Changes may have been made to this work since it was submitted for publication. A definitive version was subsequently published in Social Science \& Medicine, volume 167, in 2016. DOI:10.1016/j.socscimed.2016.08.026

The Creative Commons license below applies only to this version of the article.

\section{Creative Commons License}

\section{(c) 1 (1) 90}

This work is licensed under a Creative Commons Attribution-Noncommercial-No Derivative Works 4.0 License.

\section{Copyright}

Elsevier 
Decomposing trends in adult body mass index, obesity, and morbid obesity, 1971-2012

\begin{abstract}
Trends in adult obesity have been used to motivate key public health policies in the United States. While these analyses provide important insights into the broad historical contours of the obesity epidemic in the U.S., they shed less light on the proximate mechanisms that have generated these changes and that will ultimately determine the long-term course and pace of change in obesity rates. We used data from the National Health and Nutrition Examination Survey (NHANES), Glenn Firebaugh's linear decomposition technique, and Kitagawa's algebraic decomposition method to decompose change in body mass index (BMI), obesity, and morbid obesity from 1971 through 2012 for adults aged 20+. We partitioned change into that attributable to (1) older, fitter cohorts in the population being replaced by newer, less fit cohorts (intercohort change), and (2) cohort members becoming less fit over time (intracohort change). We found that the rise in mean BMI and rates of obesity and morbid obesity was primarily a consequence of intracohort change driven by variation in the demographic and socioeconomic composition and in the diet of the population over time. Obesity and BMI in the population rose largely because of individual increases in weight status that were broadly distributed across age and cohort groups. Cohort replacement reinforced and amplified intracohort change over the study period, leading to rapid increases in mean BMI and obesity. Because intracohort change has been the central force in the increase in BMI and obesity, successful social, dietary, medical, or policy interventions have the potential to quickly slow or reverse the upward trend in weight status. Our results also imply that policy efforts and health interventions should be broadly targeted at all age groups and birth cohorts because increases in obesity have been widely distributed across all ages and generations.
\end{abstract}

Key Words: Adults, Obesity, Body-Mass Index, Community Health, Health Policy 
Decomposing Trends in Adult BMI and Obesity

\section{Introduction}

Over the past 40 years, key public health policies have been driven by the historically unprecedented increase in adult obesity. ${ }^{1,2,3,4,5}$. In 1980, the US Public Health Service used trend data to identify obesity as a major public health concern. Armed with this information, a comprehensive plan involving both federal and state agencies was developed to combat the increased prevalence of obesity. As recently as 2015, policy-makers have used trend studies to justify the implementation of programs to educate the public on dietary and fitness recommendations that minimize obesity risk. ${ }^{6}$ Despite these efforts, the prevalence of obesity in the United States continues to increase among adults. ${ }^{7,8,9,10,11}$ The most recent data indicate that nearly one-third of adults are obese, and another $5 \%$ are morbidly obese. ${ }^{12}$

Generally, researchers attribute the increase in obesity to changes in dietary patterns and physical activity levels. ${ }^{13}$ Overlooked in these studies is the fact that changes in diet and physical activity levels can affect the obesity rate in two different ways. On one hand, the obesity rate can rise because more recent birth cohorts have poorer diets and are less physically active than previous cohorts (intercohort change or cohort replacement). On the other hand, the obesity rate can increase because the health habits of all cohorts worsen over time (intracohort change).

The magnitude and relative prominence of these two mechanisms of change have important implications for both the rate of growth and the prospect of the upward trend in obesity leveling off or reversing. For instance, if the increase in obesity largely reflects intercohort change (i.e., newer, less healthy cohorts replacing older, healthier cohorts), we would expect gradual increases in obesity over time, with obesity concentrated in more recent birth cohorts. Conversely, we would expect change resulting primarily from intracohort change (i.e., obesity rising over time for all cohorts) to occur more quickly and to be distributed more broadly among 
all birth cohorts and age groups. If cohort replacement and intracohort change reinforce one another, the obesity rate would increase rapidly over a relatively short period of time.

Disentangling the underlying mechanisms that contribute to the obesity epidemic will help researchers better understand why obesity rates continue to rise. It will also aid policymakers to develop policies and educational campaigns aimed at reducing obesity. For example, if obesity increased largely because of intercohort change, health education campaigns should be directed primarily at younger adults. On the other hand, if the rise in obesity resulted from intracohort change such efforts should be targeted more broadly.

We use data from the National Health and Nutrition Examination Survey (NHANES) to decompose change in body mass index (BMI), obesity, and morbid obesity from 1971 through 2012 for adults aged 20 years and older. We partition change into parts attributable to (1) older, fitter cohorts in the population being replaced by newer, less fit cohorts (between-cohort change), and (2) cohort members becoming less fit over time (within-cohort change).

\section{Methods}

\section{Data Sources}

Analyses are based on ten waves (1971/75, 1976/80, 1988/94, 1999/00, 2001/02, 2003/04, 2005/06, 2007/08, 2009/10, 2011/12) of the NHANES, a cross-sectional survey of the civilian, non-institutionalized U.S. population ${ }^{14}$. NHANES uses a complex multistage probability sampling design, with oversampling of smaller racial/ethnic subgroups. Analyses were estimated weighted to account for differences in the chances of selection and non-response. 
Decomposing Trends in Adult BMI and Obesity

\section{Study Population}

Models were restricted to adults 20 years and older with a completed body measurement component. Pregnant females were excluded. The total sample size for all years is 59,627 participants.

\section{Outcome Variables}

We analyzed three independent outcome measures: BMI, obesity, and morbid obesity. BMI was calculated as weight $(\mathrm{kg})$ / height $(\mathrm{m})^{2}$. We logged BMI to adjust for the non-linearity (positive skew) of BMI. Obesity was defined as a BMI $\geq 30$ and $\leq 39.9 \mathrm{~kg} \mathrm{~m}^{-2}$, and dichotomized to indicate whether or not the individual is obese. Morbid obesity was defined as a BMI at or above $40.0 \mathrm{~kg} \mathrm{~m}^{-2}$, and treated as a binary variable to indicate whether or not the individual is morbidly obese.

\section{Covariates}

We included covariates to represent demographic, socioeconomic, nutritional, and physical activity factors known to be associated with weight-related outcomes. Demographic and socioeconomic characteristics include survey year, birth year, gender ( 1 = female), race (1= nonHispanic white), marital status (1= not married), household income, and educational attainment ( 1 = less than high school). Nutrition characteristics include total intake of energy $(1=>2,000$ kcals), fat ( $1=>78$ grams), carbohydrates $(1=<100$ grams $)$, protein $(1=>56$ grams $)$, and sodium ( 1 =>1,500 milligrams) during a 24-hour period. Physical activity was classified by rate of energy expenditure $\left(1=<3\right.$ metabolic equivalents (MET) defined as $3.5 \mathrm{ml} \mathrm{O}_{2} \cdot \mathrm{kg}^{-1} \cdot \mathrm{min}^{-1}$ ) pursuant to a particular activity. 
Decomposing Trends in Adult BMI and Obesity

\section{Statistical Analysis}

We used Glenn Firebaugh’s (1989) linear decomposition technique to decompose aggregate (i.e., total) change in weight-related outcomes from 1971 through 2012. With this approach, cohort differences were estimated by regressing obesity on birth year and survey year. This approach assumed linearity. Because change may not be linear, we also collapsed birth year into cohort subgroups ${ }^{15}$, and used Kitagawa's algebraic decomposition method that does not assume linearity ${ }^{16}$. Both methods allowed us to partition total change (TC) in weight-related outcomes into two components of aggregate change: intracohort change (IC) and cohort replacement (CR). Intracohort change is computed by multiplying the regression coefficient for the survey year variable by the length of the study period (i.e., last survey year - first survey year). Cohort replacement is computed by multiplying the regression coefficient for the birth year variable by the difference between the mean birth years for the final and initial survey years. IC indicates how much of the aggregated change in weight status is attributable to individual change in body weight (i.e., period changes), and CR tell us how much of the total change in weight status is attributable to population turnover (i.e., the death of old cohorts with lower BMIs and the birth of new cohorts with higher BMIs). We assigned the midpoint mean for each survey wave (e.g., if 1971/73 we used 1972) and used linear interpolation methods to account for the multi-year data collection design in the NHANES data.

\section{Results}

BMI, Obesity, and Morbid Obesity increased significantly between 1971 and 2012 (Table 1). In 1971, the average BMI for adults aged 20 years and older was 25.66 (logged = 3.23). By 2012, the mean BMI increased to 28.71 (logged = 3.33). Obesity increased by nearly 15 
Decomposing Trends in Adult BMI and Obesity

percentage points from 1971 to 2012, whereas morbid obesity rose by 5 percentage points during this period.

Figures 1-3 display historical trends in BMI, obesity, and morbid obesity, overall and for men and women, between the early 1970s and the early 2010s. Between the early and the late 1970s, mean BMI and obesity rates were remarkably stable. Beginning in the early 1980s, both average BMI and obesity rates rose sharply. Average BMI rose by $9 \%$ between the early 1980s and the late 1990s, with mean BMI increasing from 25.23 in the 1976/80 wave to 27.52 in the 1999/2000 wave. Over this period, the obesity rate nearly doubled, rising from $13 \%$ to $22 \%$, and the morbid obesity rate quadrupled, from $1 \%$ to $4 \%$. Increases in BMI and obesity have moderated considerably since the late 1990s.

$<$ Figures 1, 2 , \& 3>

Obesity and morbid obesity rates (but not mean BMIs) are somewhat higher for women than for men from the early 1970s into the 1990s. For example, the obesity rate for women in the $1971 / 74,1976 / 80$, and 1988/90 waves average about $18 \%$ and the morbid obesity rate averaged about $3 \%$, whereas the obesity rate for men averaged about $14 \%$ and the morbid obesity rate averaged about 1\%. While trends in BMI and obesity for men and women parallel one another, obesity rates for men grew more sharply than they did for women. For both men and women, mean BMI and obesity rates were largely unchanged between 1971/74 and 1976/80. Between 1976/80 and 1999/2000, the obesity and morbid obesity rates for men increased by $83 \%$ and 326\%, respectively, whereas these rates grew by 58\% and $161 \%$ for women. Since 1990/2000, the obesity rates for men and women rose by $35 \%$ and $28 \%$, whereas the morbid obesity rate for both men and women increased by about $60 \%$. 
Decomposing Trends in Adult BMI and Obesity

The sharp increase in BMI and obesity over the last 40 years may reflect two very different processes of change. On one hand, obesity and BMI may have increased because population turnover has led to the replacement of older, lower BMI, and lower obesity rate birth cohorts by more recent, higher-BMI, and higher obesity rate cohorts. Conversely, BMI and obesity rates for all birth cohorts may have risen over time as a consequence of individual increases in BMI and obesity over time.

Before partitioning total change into its IC and CR components, we present mean BMI, obesity, and morbid obesity rates by age for the cohorts in our study with sufficient sample sizes $(\mathrm{N} \geq 50)$ for a minimum of three of the ten-year age group intervals (Figures 4-6). If IC is central to the increase in BMI and obesity, BMI and obesity rates for cohorts will rise as their members age. If, on the other hand, CR is crucial to this increase, BMI levels and obesity rates for more recent cohorts will be higher than for previous cohorts throughout adulthood. If both IC and CR are important, not only will BMI and obesity rates be higher for more recent cohorts but BMI and obesity will rise as cohort members age.

$<$ Figures 4, 5, 6>

Figures 4-6 show that both IC and CR played significant roles in the increase in BMI and obesity over the last 40 years. Consistent with the CR explanation, more recent cohorts have higher BMIs and obesity and morbid obesity rates than earlier cohorts over the course of adulthood. For example, 8\% of 20-29 year olds born between 1940 and 1949 were obese (1\% were morbidly obese), while 17\% of 20-29 year olds born between 1970 and 1979 were obese (3\% were morbidly obese). At the other end of the age spectrum, the obesity rate for 70 to 79 year olds rose from $18 \%$ for the $1910-1919$ cohort to $35 \%$ for the $1940-1949$ cohort, and the morbid obesity rate for this age group increased from 3\% to 8\%. Consistent with the IC 
explanation, BMI and obesity rates also increase over the course of adulthood for nearly all cohorts. For example, the obesity and morbid obesity rates for the 1940 to 1949 cohort rose from $8 \%$ and $1 \%$, respectively, at ages $20-29$ to $35 \%$ and $8 \%$ at ages $70-79$. With the exception of one of the earliest cohorts (1910-1919), BMI, obesity, and morbid obesity increased sharply as cohort members aged over time.

Figure 7 displays the percentages of total change in BMI, obesity, and morbid obesity due to IC and CR for unadjusted models (no control variables), and for conditional models that adjust for demographic, socioeconomic, nutritional, and physical activity differences among cohorts and time periods. Figure 7 shows that the sharp increases in mean BMI and in the obesity and morbid obesity rates in the U.S. over the last four decades largely reflect within-cohort change (i.e., individual change). The top bar in each panel in Figure 7 displays the percentages of overall change in BMI, obesity, and morbid obesity, respectively, attributable to IC (dark gray) and CR (light gray), if compositional differences among the cohorts are ignored. Individual, withincohort change, accounts for $77 \%$ of total change in logged BMI, $76 \%$ of overall change in the obesity rate, and $63 \%$ of aggregate change in morbid obesity. Only one-quarter to about onethird of the overall increase in BMI and obesity resulted from earlier, lower BMI, and less obese birth cohorts being replaced by more recent, higher BMI, and more obese cohorts. BMI, obesity, and morbid obesity rates rose mainly because the typical member of all cohorts became heavier over time. Results from the algebraic decomposition method, which does not assume linearity, also show that the aggregate increases in weight status over the last four decades principally reflect intracohort change.

$<$ Figure $7>$ 
Decomposing Trends in Adult BMI and Obesity

To better understand the sources of aggregate change in weight status, we also computed TC, IC and CR for models that control for demographic and socioeconomic, nutritional, and physical activity changes in the population over time. Figure 8 compares estimates of total change in BMI and obesity and morbid obesity rates for the unconditional model that does not control for compositional changes to a series of models that adjusted for demographic and socioeconomic, nutritional, and physical activity differences among the cohorts and time periods. Compositional changes in demographic composition and socioeconomic status, nutrition, and physical activity by-and-large do not explain the increase in weight status over the study period. Controlling for all factors (bottom bar in each panel in Figure 8) reduced TC in logged BMI from 0.189 to 0.144 , in the obesity rate from 0.249 to 0.242 , and in the morbid obesity rate from 0.079 to 0.047. Adjusting for demographic and socioeconomic, nutritional, and physical activity changes in the population over time accounts for $23.8 \%$ of the increase in logged BMI, $2.8 \%$ of the rise in obesity, and $40.5 \%$ of the uptick in morbid obesity.

$<$ Figure $8>$

Accounting for changes in the population composition may affect the relative contributions of individual change (i.e., IC) and population turnover (i.e., CR) to aggregate change (i.e., TC). For example, if more recent cohorts had higher BMIs and obesity rates than did previous cohorts because they were less active and had poorer diets, controlling for changes in physical activity and diet would attenuate or eliminate the influence of CR on TC. The $2^{\text {nd }}$ through $5^{\text {th }}$ bars in each panel in Figure 7 show how adjusting for these compositional differences influenced the relative contributions of IC and CR. If we compare the top bars in each panel (i.e., no controls) and bottom bars in each panel (i.e., all controls) in Figure 7, we see that when we account for the demographic and socioeconomic, nutritional, and physical activity 
Decomposing Trends in Adult BMI and Obesity

differences among cohorts the estimated contribution of cohort replacement to the increase in body weight is greatly reduced. After adjusting for compositional differences among the cohorts, CR did not contribute to the increase in logged BMI (<0\%), contributed minimally to the rise in obesity (4.0\%), and contributed moderately to the increase in morbid obesity (20.0\%). For logged BMI and the obesity rate, the modest influence of CR we observed for the unconditional model reflects between-cohort differences in demographic and socioeconomic characteristics, physical activity levels, and diet. For the morbid obesity rate, the larger contribution of CR we found (33\% vs 20\%-25\%), only partially reflects these between-cohort compositional differences.

To identify which compositional differences between cohorts were most important, we computed TC, IC, and CR for models that entered demographic and socioeconomic factors, physical activity levels, and dietary measures separately. The middle three bars in each panel in Figure 8 display estimated TC for these models, and the middle three bars in each panel in Figure 7 display estimated IC and CR for these models. Adjusting for differences in physical activity levels in the population over the study period had a negligible effect on estimates of TC, IC, and CR for the three weight status measures, although adjusting for activity levels did reduce estimated total change in logged BMI by about 15\%. Changes in physical activity levels were not responsible for higher obesity rates over the study period, nor were between-cohort differences in obesity attributable to differences in physical activity levels. Changes in diet and nutrition, and in the demographic and socioeconomic composition of population, played a more prominent role in the rise in obesity and in intercohort differences in obesity. Changes in nutrition and diet account for $16 \%$ of the overall increase in obesity and $20 \%$ of the rise in logged BMI, whereas changes in the demographic and socioeconomic composition of population account for more than one-third 
Decomposing Trends in Adult BMI and Obesity

of TC in morbid obesity and nearly one-fifth of TC in logged BMI. BMI and obesity rates rose in part because diet and nutrition worsened over time, and because the size of demographic and socioeconomic status groups with higher BMIs grew and the size of groups with lower BMIs contracted. Adjusting for between-cohort differences in demographic and socioeconomic composition, and in nutrition and diet, also substantially reduced estimates of CR. More recent cohorts have higher BMIs and obesity rates than earlier cohorts because they are less demographically and socioeconomically advantaged, and because they have poorer nutrition and diets.

\section{Discussion}

The obesity rate in the U.S. has doubled since the 1970s and the morbid obesity rate has increased nearly fivefold (Figures 2 and 3). This rapid and largely unanticipated increase in obesity rates has led public health officials to declare an “epidemic of obesity in the U.S.” 17 Understanding the demographic mechanisms that have generated these changes help us to better understand the reasons for this precipitous increase in obesity and to more effectively anticipate the long-term course and pace of change in obesity rates. If, for example, increases in obesity largely reflect population turnover, we would expect obesity rates to rise gradually over time as new, more obese birth cohorts replace older, less obese cohorts. If, on the other hand, change is the result of intracohort change, we would anticipate obesity rates will increase more quickly as all birth cohorts and age groups experience increases in obesity. If intracohort change and cohort replacement mechanisms reinforce one another, the obesity rate will rise rapidly over a relatively short period of time. ${ }^{18}$

We find that the rise in mean BMI and rates of obesity and morbid obesity among adults aged 20 years and older was primarily a consequence of intracohort change. Obesity and BMI in 
the population rose largely because of individual increases in weight status that were broadly distributed across age and cohort groups. Smaller but significant cohort replacement reinforced and amplified these intracohort change differences over the study period, leading to rapid increases in mean BMI and obesity between 1971 and 2012.

Obesity rates rose, in part, because of changes in the demographic and socioeconomic composition and in the diet of the population over time. The obesity and morbid obesity rates increased because the typical adult in 2012 consumed more calories, more protein, more fat, and more sodium than the typical adult in 1971 and because the size of demographic and socioeconomic status groups with higher BMIs such as the poor grew and the size of groups with lower BMIs contracted between 1971 and 2012. Changes in the demographic and socioeconomic composition, diet and nutrition, and physical activity levels in the population over time accounted for only a small portion of the increase in obesity (2.8\%), a modest share of the increase in logged BMI (24.0\%), and sizable of the increase in morbid obesity (40.5\%).

In a climate of unhealthy, processed food, sedentary labor, and limited capacity of policymakers to implement changes in the absence of strong public support for policy action, we are faced with numerous barriers to reversing the upward trend in obesity in the United States. ${ }^{19,20,21}$ Our results, however, provide some reasons for optimism that growth in obesity might be slowed or reversed. Because individual change (i.e., intracohort change) has been the central force in the increase in BMI and obesity, successful social, dietary, medical, or policy interventions have the potential to quickly slow or reverse the upward trend in weight status. Our results also imply that policy efforts and health interventions should be broadly targeted at all age groups and birth cohorts because increases in obesity have been widely distributed across all ages and generations. 


\section{References}

1. Novak NL, Brownell KD. Role of Policy and Government in the Obesity Epidemic. Circ. 2012; 126: 2345-2352.

2. Malik VS, Willett WC, Hu FB. Global Obesity: Trends, Risk Factors and Policy Implications. Endocrinol. doi:10.1038/nrendo.2012.199.

3. Gortmaker SL, Swinburn BA, Levy D, et al. Changing the Future of Obesity: Science, Policy, and Action. Lancet 2011; 378: 838-47.

4. Huang TT-K, Cawley JH, Ashe M, et al. Mobilisation of Public Support for Policy Actions to Prevent Obesity. Lancet 2015; http://dx.doi.org/10.1016/S0140-6736(14)61743-8.

5. Institute of Medicine. Accelerating Progress in Obesity Prevention: Solving the Weight of the Nation. Washington, DC: National Academies Press; 2012.

6. Levi J, Rayburn J, Segal LM, Martin A. The State of Obesity: Better Policies for a Healthier America. Princeton, NJ: Robert Wood Johnson Foundation; 2015.

7. Ogden CL, Carroll MD, Flegal KM. Prevalence of Obesity in the United States. JAMA. 2014; 312(2):189-190. doi:10.1001/jama.2014.6228.

8. Ogden CL, Carroll MD, Kit BK, Flegal KM. Prevalence of Obesity and Trends in Body Mass Index Among US Children and Adolescents, 1999-2010. JAMA. 2012; 307(5):483-490. doi:10.1001/jama.2012.40.

9. Flegal KM, Carroll MD, Ogden CL, Curtin LR. Prevalence and Trends in Obesity Among US Adults, 1999-2008. JAMA. 2010; 303(3):235-241. doi:10.1001/jama.2009.2014.

10. Ogden CL, Carroll MD, Curtin LR, McDowell MA, Tabak CJ, Flegal KM. Prevalence of Overweight and Obesity in the United States, 1999-2004. JAMA. 2006; 295(13):1549-1555. doi:10.1001/jama.295.13.1549.

11. Flegal KM, Carroll MD, Ogden CL, Johnson CL. Prevalence and Trends in Obesity Among US Adults, 1999-2000. JAMA. 2002; 288(14):1723-1727. doi:10.1001/jama.288.14.1723.

12. Center for Disease Control. Health, United States, 2015, table 53. http://www.cdc.gov/nchs/fastats/obesity-overweight.htm. Accessed February 21, 2016. 
13. Lim SS, Vos T, Flaxman AD, et al. A Comparative Risk Assessment of Burden of Disease and Injury Attributable to 67 Risk Factors and Risk Factor Clusters in 21 Regions, 1990-2010: A Systematic Analysis for the Global Burden of Disease Study 2010. Lancet 2012; 380: 2224-60.

14. CDC National Center for Health Statistics. National Health and Nutrition Examination Survey. 2011; http://www.cdc.gov/nchs/nhanes/nhanes_questionnaires.htm. Accessed February 1, 2016.

15. Firebaugh G. Methods for Estimating Cohort Replacement Effects. Sociol Methodol 1989; 19: $243-262$.

16. Kitagawa E. Components of a Difference between Two Rates. J Am Stat Assoc. 1955;

50:1168-1194.

17. Obesity Declared an Epidemic in the United States. Journal of the National Medical Association 1999; 91(12): 645.

18. Wagmiller RL, Schultz Lee K. Are Contemporary Patterns of Black Male Joblessness Unique? Cohort Replacement, Intracohort Change, and the Diverging Structures of Black and White Men’s Employment. Soc Probl. 2014; 61(2): 305-327.

19. Roberto CA, Swinburn B, Hawkes C, Huang TTK, Costa SA, Ashe M, et al. Patchy Progress on Obesity Prevention: Emerging Examples, Entrenched Barriers, and New Thinking. Lancet 2015; 385 2400-2409.

20. Dhurandhar,E J. The downfalls of BMI-focused policies. Int J Obes 2016; 40 (5): 729-730 http://dx.doi.org/10.1038/ijo.2016.1810.1038/ijo.2016.18.

21. Liu, S.Y., Walter, S., Marden, J., Rehkopf, D.H. Kubzansky, Nguyen, L.D. T, and M.M. Glymour. 2014. Genetic vulnerability to diabetes and obesity: Does education offset the risk? Social Science \& Medicine 127: 150-158. doi:10.1016/j.socscimed.2014.09.009 


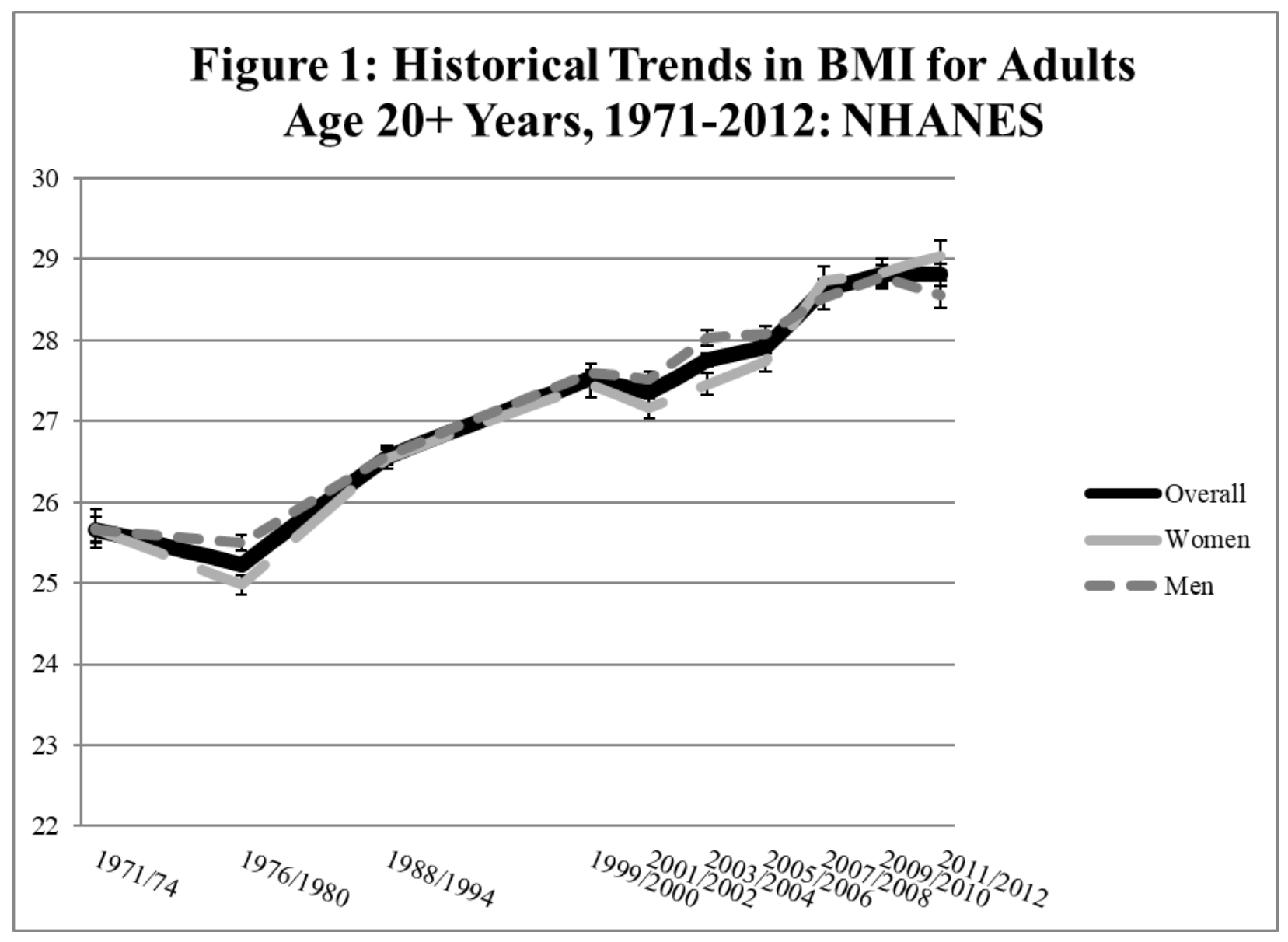




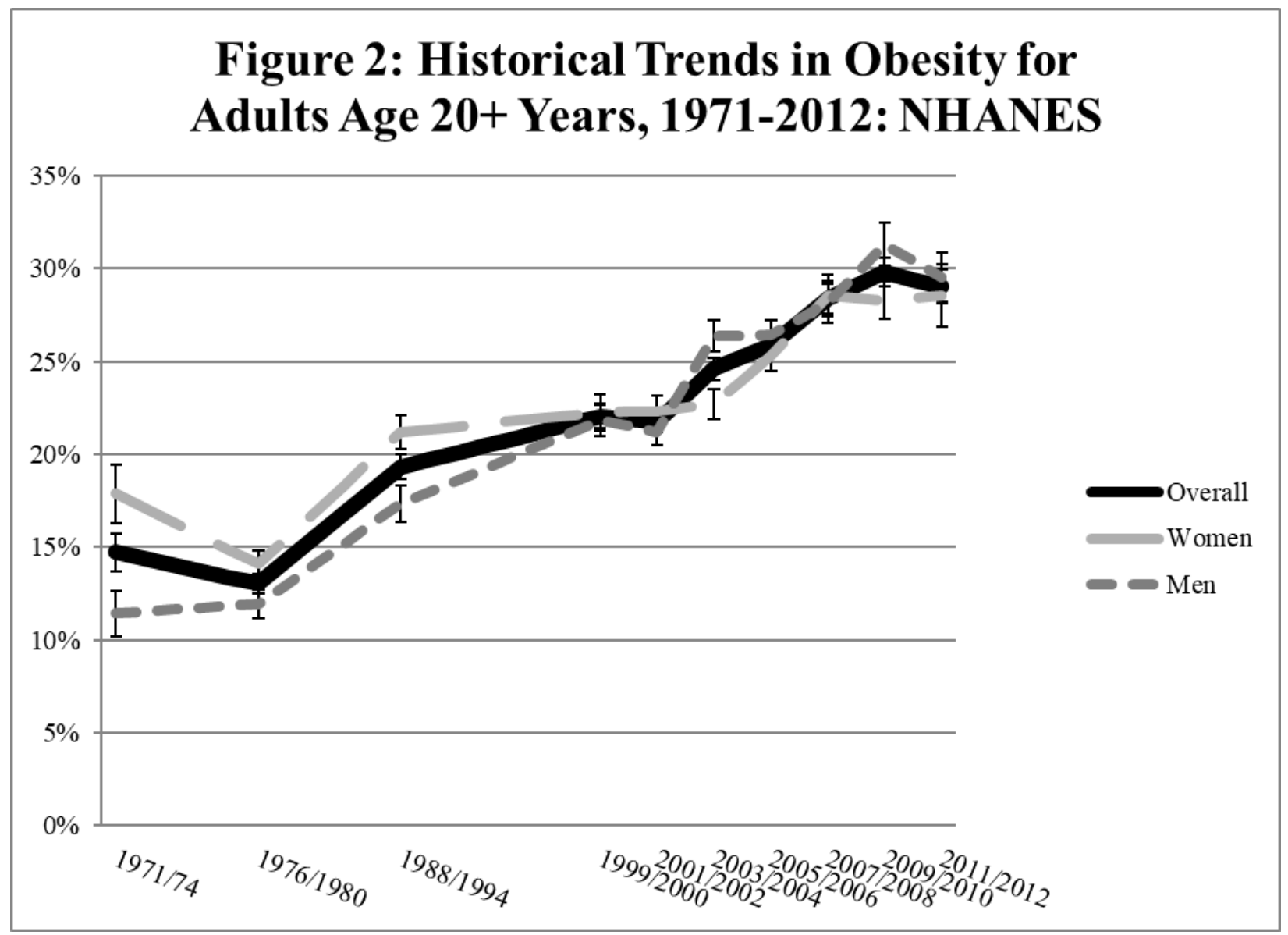




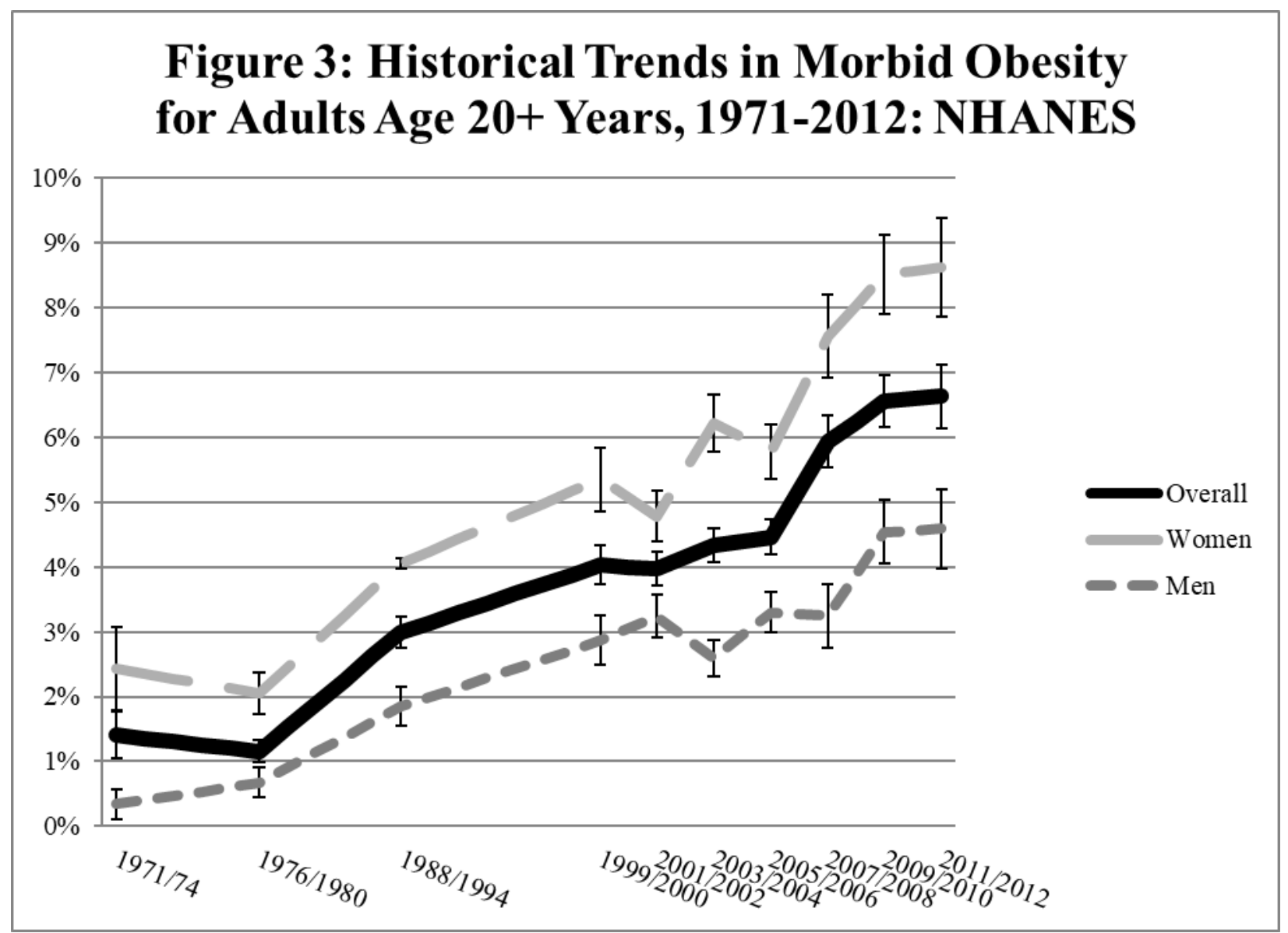




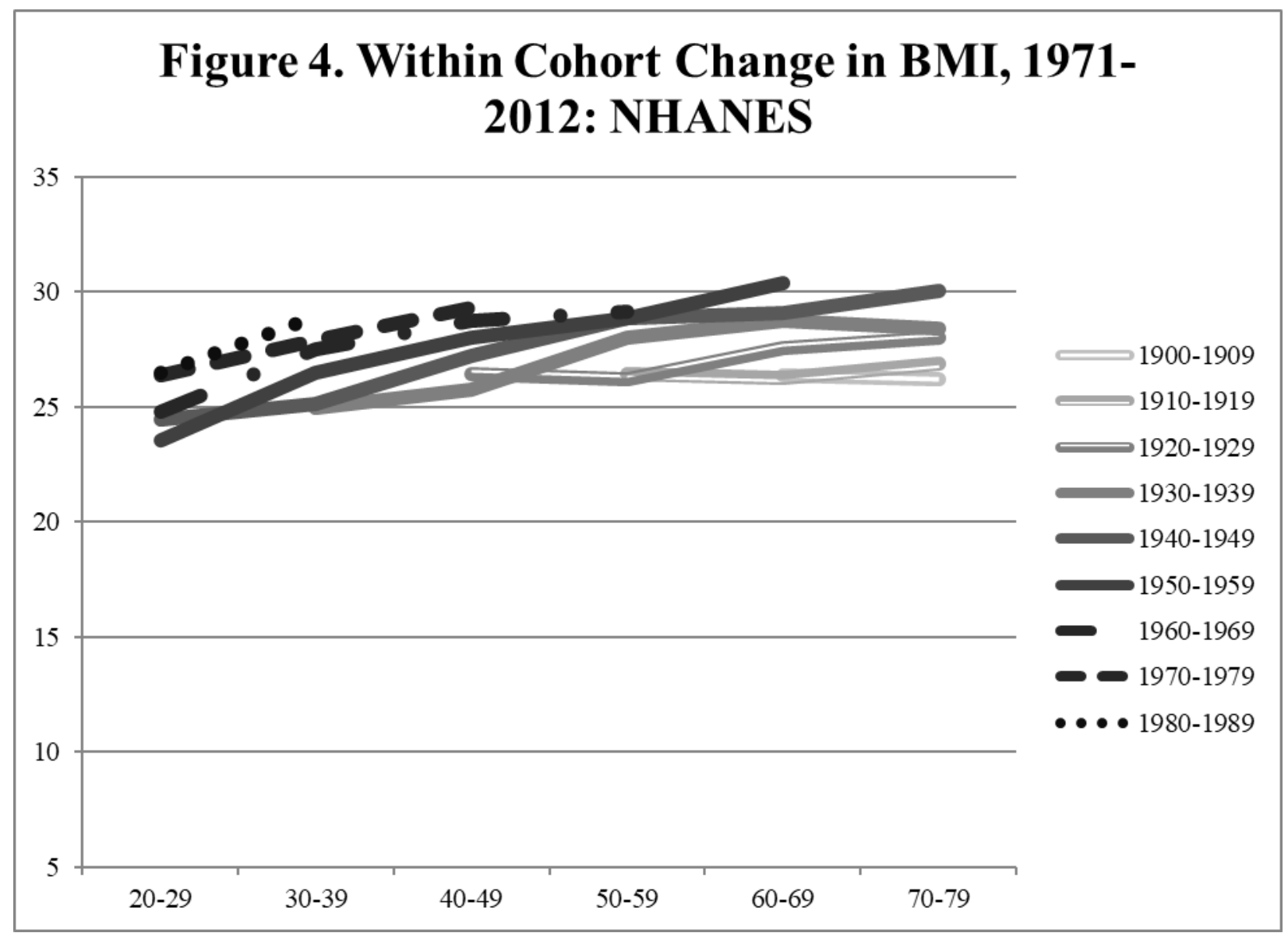




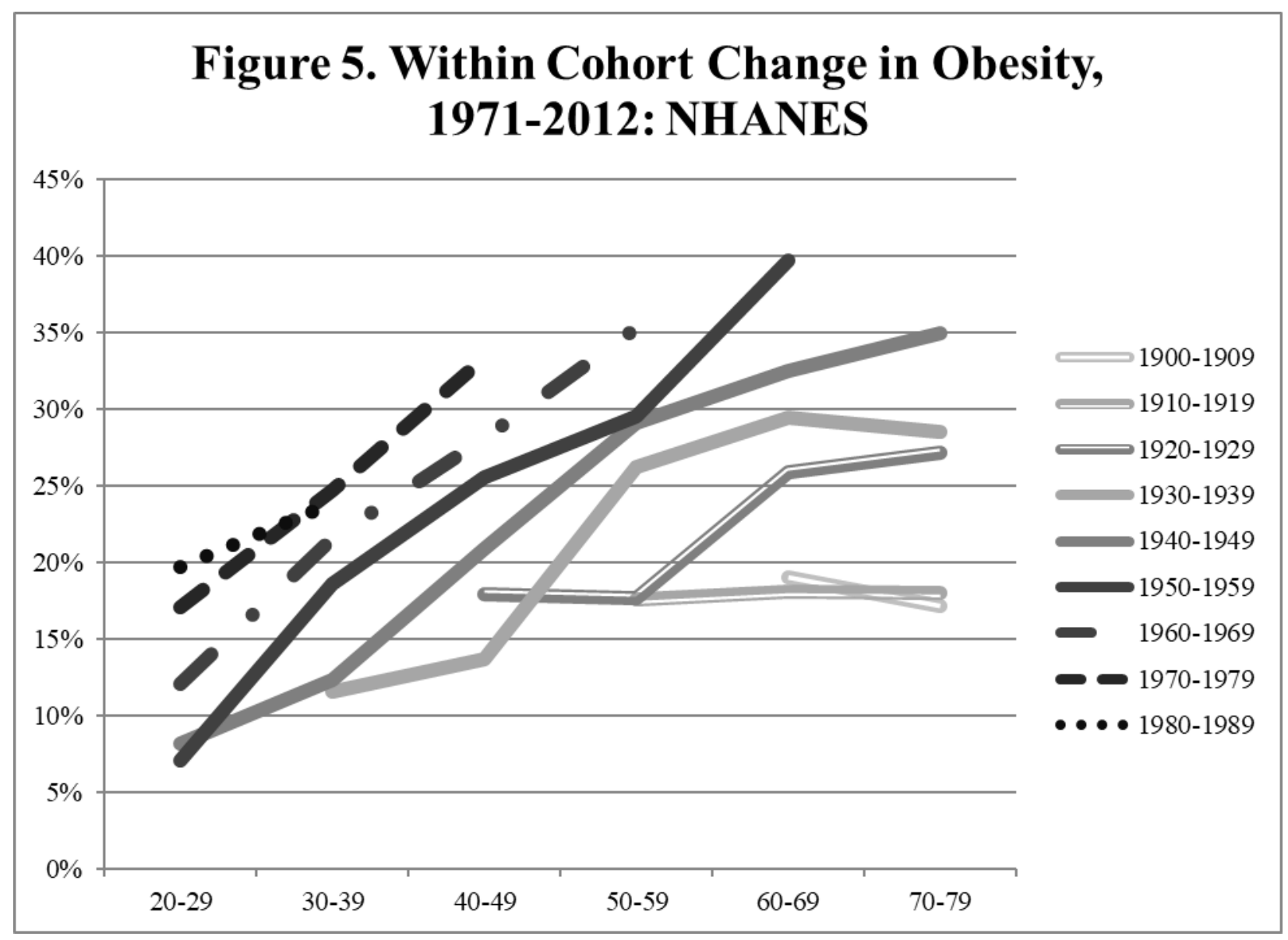




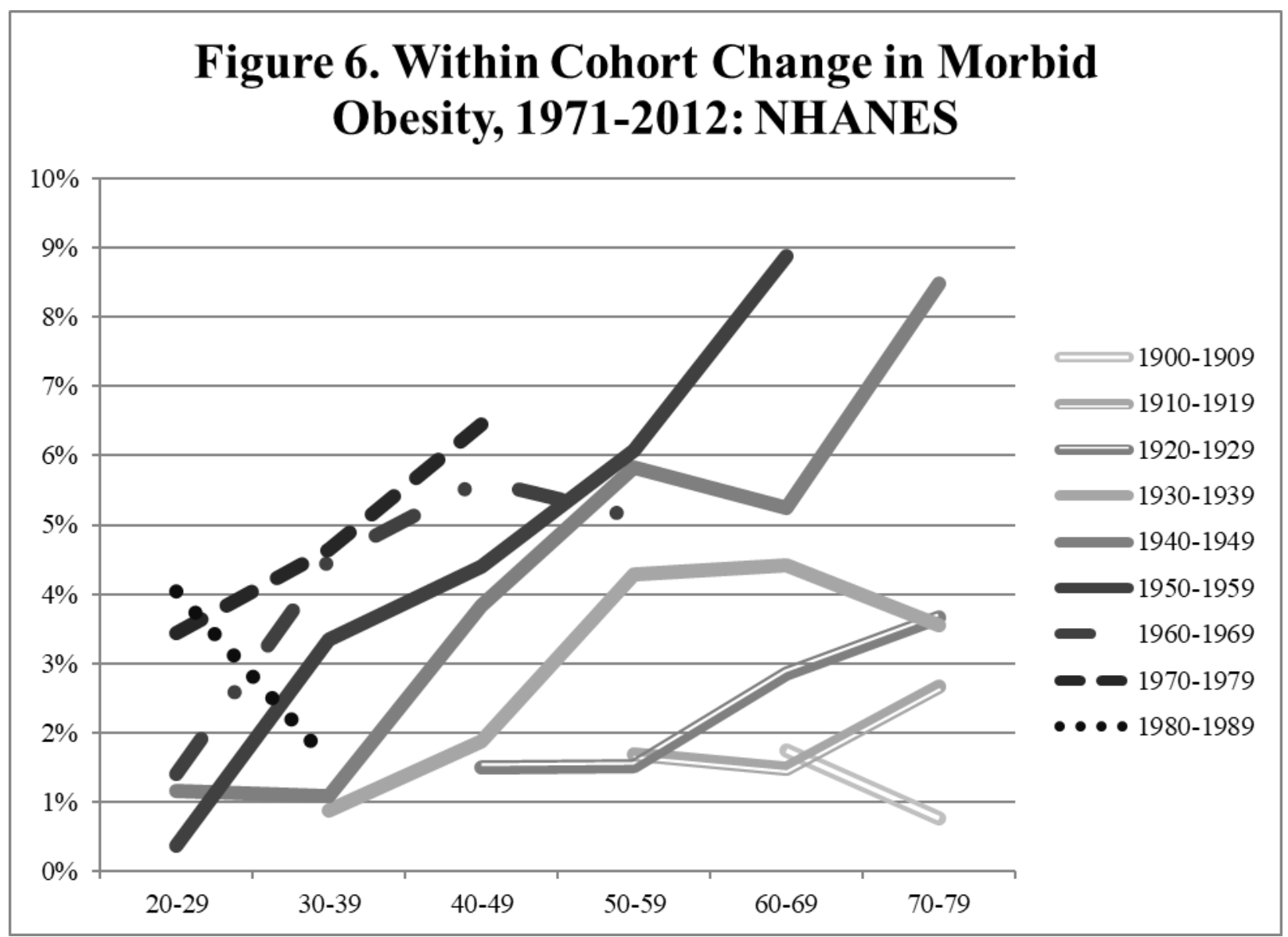




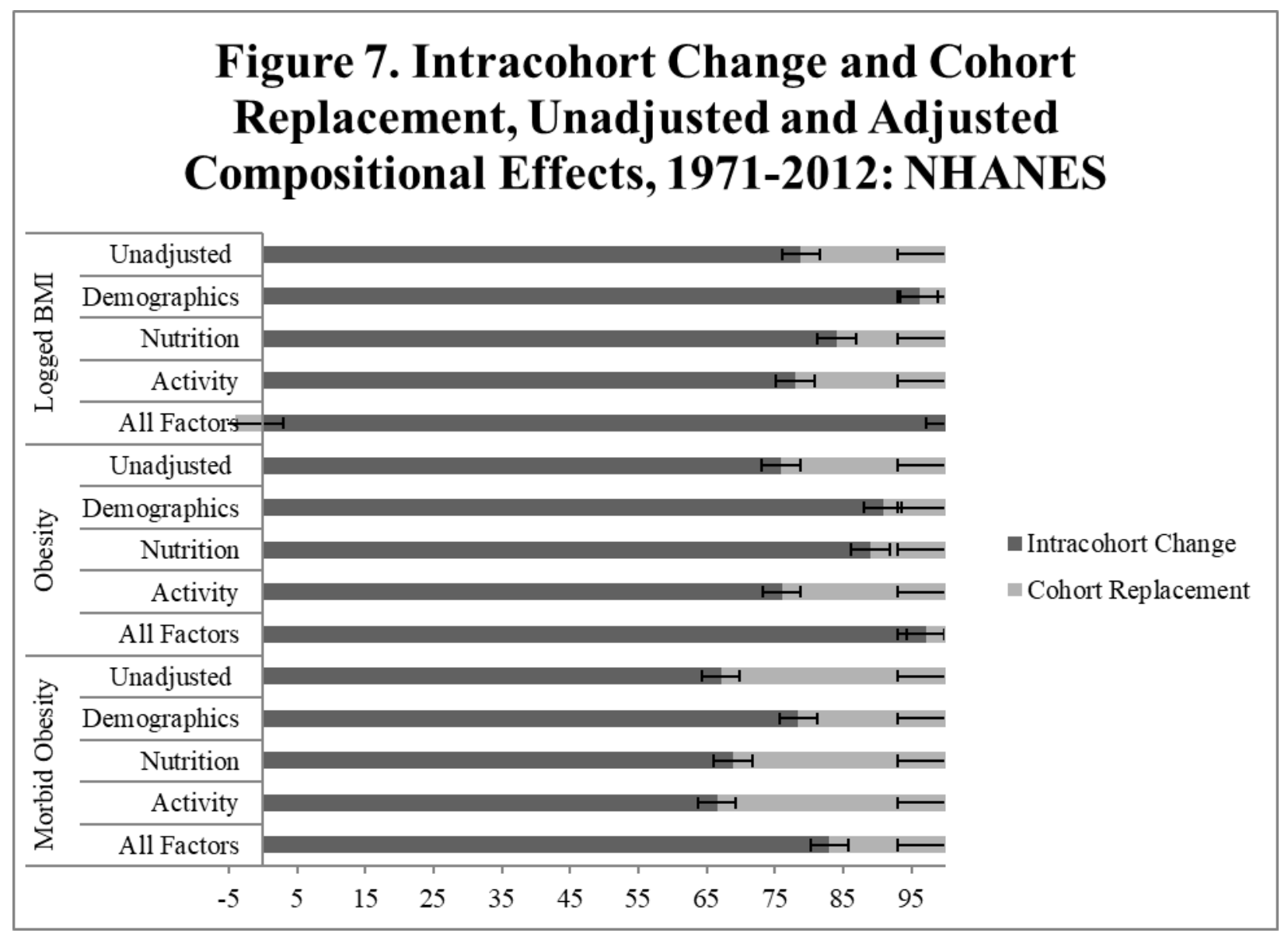




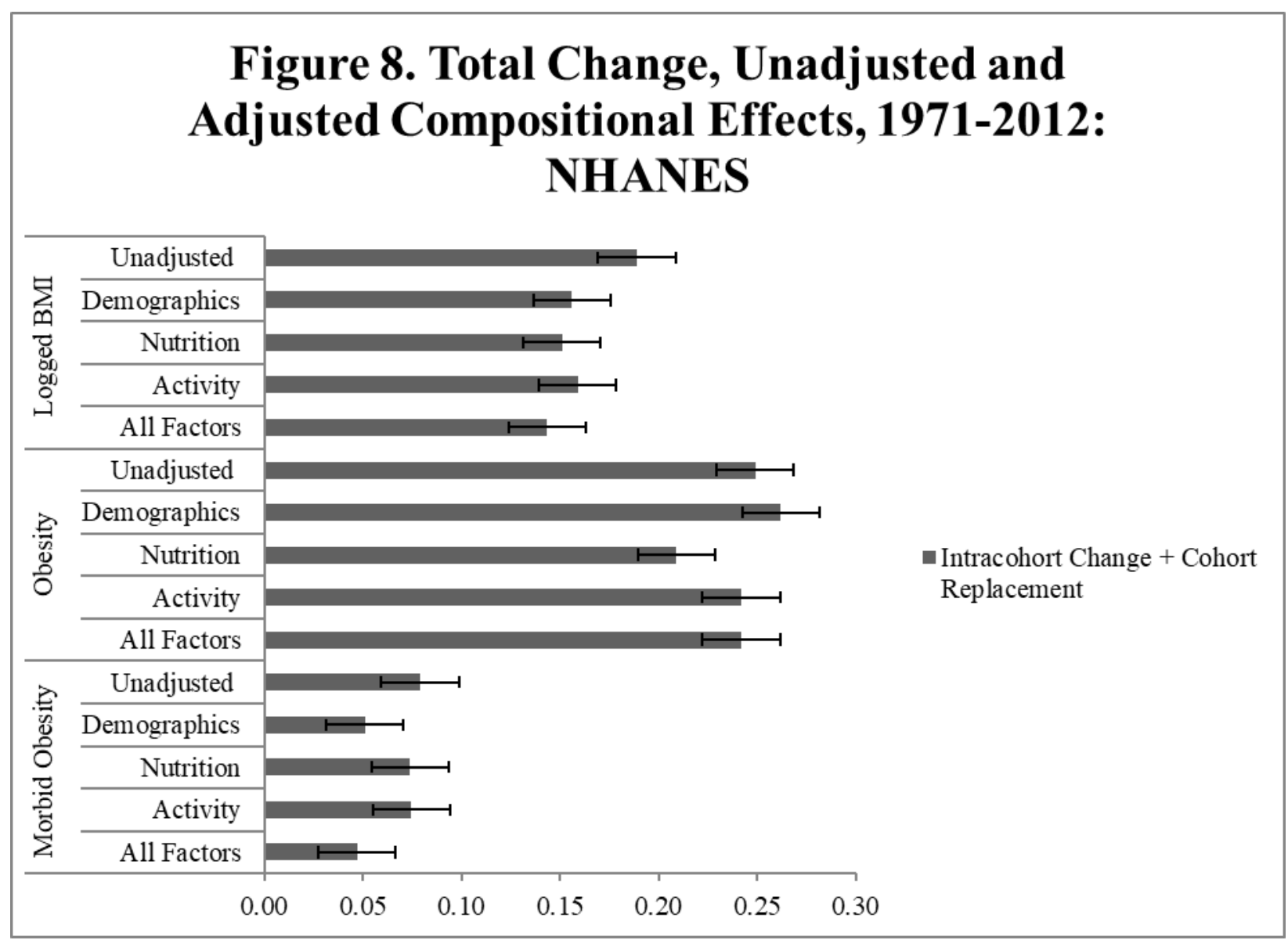

